\title{
THE STARFISH EXPERIMENT: A LAGRANGIAN APPROACH
}

\author{
A. Sneyd ${ }^{1}$, J. Etay ${ }^{2}$, Y. Fautrelle ${ }^{2}$ \\ ${ }^{1}$ Dept. of Mathematics, University of Waikato, Private Bag, Hamilton, New Zealand \\ ${ }^{2}$ EPM-Madylam/INPG/CNRS, ENSHMG, \\ BP 95, 38402 Saint Martin d'Héres Cedex, France
}

The present paper analyses the free surface deformation of a liquid metal drop under the influence of an alternating magnetic field. The analysis is restricted to the first axisymmetric mode oscillation. In the low frequency case, the electromagnetic forces are of gradient type and purely oscillatory. Without any viscous damping, it is then possible to build a Lagrangian function, which involves the kinetic energy, the gravitational energy, the surface energy and the electromagnetic energy. The time evolution of the pool height is easily obtained from the Lagrange equation. It is shown that the pool height behaves like a non-linear forced oscillator.

Introduction. When a liquid metal drop is submitted to a vertical alternating magnetic field, it tends to oscillate in a horizontal plane. This phenomenon has been put forth in the so-called "Starfish experiment" (Daugan et al. [1], Ingwiller et al. [2]). According to the magnetic field strength and its frequency $f$, various types of motions have been observed. For weak magnetic field intensities, an initially axisymmetric drop exhibits an axisymmetric oscillatory motion which is mainly vertical. This dynamical deformation is directly forced by the electromagnetic forces, and their oscillation frequency is $2 f$. Then, for stronger magnetic field values a symmetry breaking appears, and the drop oscillates in a horizontal plane with a frequency $f$. The strongest horizontal oscillations are localised near the resonance point corresponding to the natural frequencies of the drop. This regime is similar to a so-called parametric oscillation already observed in other configurations (Galpin et al. [3]). Such a phenomenon may be applied to determine the liquid tension of a liquid metal. The surface patterns obtained with a mercury drop are illustrated in Fig. 1, which shows both the axisymmetric motion

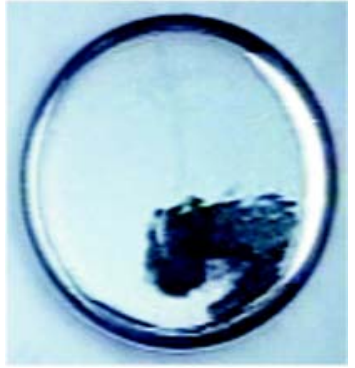

(a) $I=0$

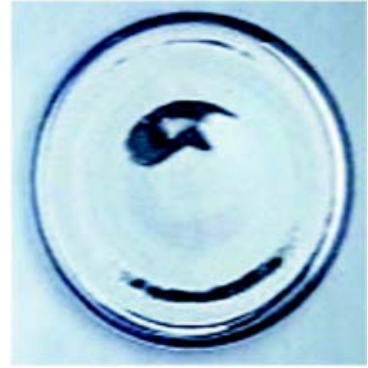

(b) $f=1.354 ; I=65 \mathrm{~A}$

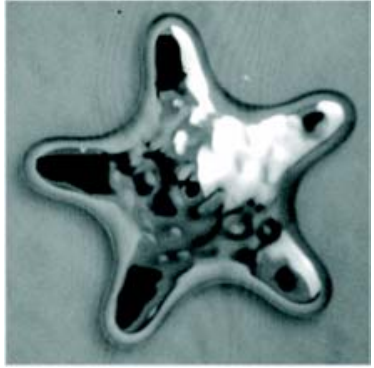

(c) $f=1.779 ; I=77 \mathrm{~A}$

Fig. 1. Free surface patterns viewed from above in the first regime for various magnetic field frequencies; the various pictures show the mode selection according to the applied frequency; $a=30 \mathrm{~mm}$ [1]; the proportionality factor between the magnetic field strength (maximum value in Tesla) and the coil intensity $I$ (r.m.s. value in Ampere) is 0.0024 ; in $(a)$ and $(b)$ the oscillation frequency is equal to twice that of the magnetic field (forcedwave regime); in (c) the oscillation frequency is equal to that of the magnetic field (parametric regime). 
as well as the azimuthal oscillations. When the coil current is zero, the drop is at rest and its shape viewed from above is circular (Fig. 1a). For a weak coil current, the drop oscillates, but its shape remains axisymmetric as shown in Fig. $1 b$. This corresponds to the forced-oscillation regime.

The aim of the present paper is to achieve at first theoretical analysis the motion of the drop in order to have a better understanding of such a phenomenon. Thanks to the particular form of the electromagnetic forces in the low frequency limit [4], we propose to use a Lagrangian approach in order to describe the drop motion. For that purpose, a simple geometrical model is built in order to describe the method. We deal with the first axisymmetric motion, which consists of the first oscillation mode. The mathematical model is described in Section 2, whilst some results are yielded in Sections 2 and 3.

1. The mathematical model. As a first attempt to model the drop, we consider an axisymmetric horizontal drop of liquid metal in the presence of a vertical alternating magnetic field

$$
\mathbf{B}=B_{0} \sin (\omega t) \mathbf{i}_{z},
$$

$B_{0}, \omega$ and $\mathbf{i}_{z}$ denoting respectively the typical applied magnetic field strength, its pulsation and a unit vector along the vertical. The geometry is sketched in Fig. 2 . We assume that the edge of the pool remains straight and parallel to the $y$-axis and that its height $h(t)$ is time dependent but uniform. The drop is supposed to be flat. Accordingly, the pool height is significantly smaller than its radius $(h<<a)$. It follows that the volume $V_{0}$ of the pool is related to its radius $a(t)$ and height $h$ by

$$
V_{0}=\pi h a^{2} \equiv \pi V=\text { constant. }
$$

We assume that the fluid is inviscid and the motion is irrotational. We consider natural oscillations of the liquid pool involving periodic variations in $h(t)$ and $a(t)$ - the pool broadening and narrowing, then becoming thinner and higher in accordance with (2). Such oscillations are described by time evolution of the single variable $h(t)$. Note that such a deformation may only represent the first axisymmetric oscillatory mode. The analytical method consists in defining a Lagrangian function, which involves the kinetic energy, surface energy, the gravitational energy

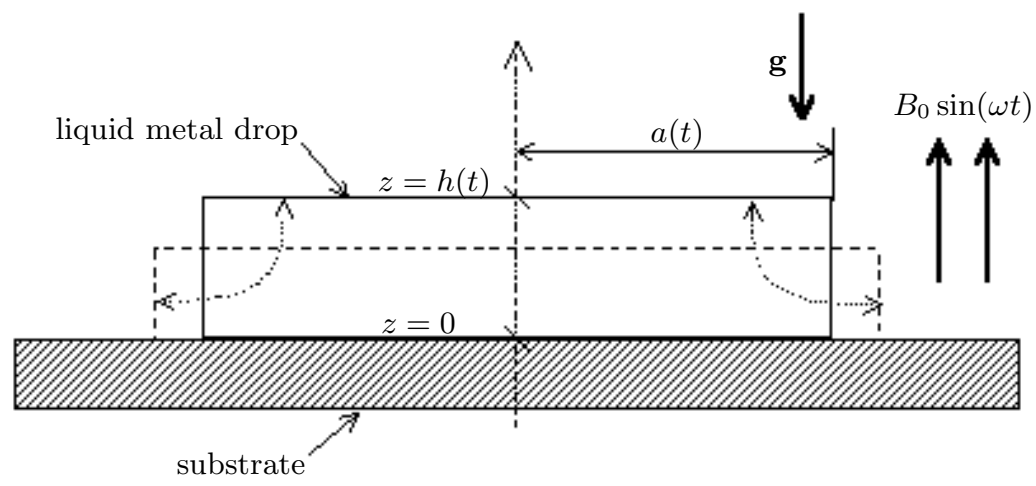

Fig. 2. Sketch of the oscillating drop. 
and the potential energy of the magnetic field. Let us evaluate each contribution separately.

1.1. Kinetic energy. Owing to the above hypotheses, the velocity field is governed by a harmonic scalar potential $\phi$ such that

$$
\phi=\frac{\dot{h}}{2 h}\left(z^{2}-\frac{r^{2}}{2}\right), \quad \text { with } \quad \dot{h}=\frac{\mathrm{d} h}{\mathrm{~d} t} .
$$

It is then possible to determine the overall kinetic energy $E_{k}(t)$ of the drop from (3) by integrating the local kinetic energy in the whole liquid domain $D$

$$
E_{k} \equiv \frac{1}{2} \int_{D} \rho(\nabla \phi)^{2} \mathrm{~d} \nu=\frac{1}{4} \rho \pi \dot{h}^{2} V\left[\frac{3}{2}+\frac{V}{4 h^{3}}\right] .
$$

1.2. Surface energy. The surface energy $E_{s}$ is readily obtained from the overall drop area and the surface tension $\gamma$

$$
E_{s}=\gamma \pi a^{2}\left(1+2 \frac{h}{a}\right) \approx \gamma \pi a^{2}
$$

1.3. Electromagnetic potential energy. In the case where the magnetic field frequency is weak, the electromagnetic forces $\mathbf{F}_{\text {em }}$ may be expressed in a simple way from the so-called torsion function $T(r)$ (see [4] for details)

$$
\mathbf{F}_{\mathrm{em}}=\frac{1}{2} \sigma \omega B_{0}^{2} \sin (2 \omega t) \nabla T
$$

In an axisymmetric geometry, the torsion function takes the following simple expression

$$
T=\frac{1}{4}\left(a^{2}-r^{2}\right)
$$

The electromagnetic force potential $\Phi_{\mathrm{em}}$ is readily obtained from (6) and (7), and the corresponding electromagnetic potential energy $E_{\mathrm{em}}$ is

$$
E_{\mathrm{em}} \equiv \int_{D} \Phi_{\mathrm{em}} \mathrm{d} \nu=\frac{\pi}{16} \sigma \omega B_{0}^{2} \sin (2 \omega t) \frac{V^{2}}{h} .
$$

1.4. Gravitational potential energy. The potential energy $E_{g}$ due to the gravity forces is obtained from the following integral

$$
E_{g} \equiv \int_{D} \rho g z \mathrm{~d} \nu=\frac{1}{2} \rho g \pi V h,
$$

$g, z$ being respectively the gravity and the vertical coordinate.

1.5. Lagrange equation. We may then define a Lagrangian function $L(h, \dot{h})$ such that

$$
L(h, \dot{h}) \equiv E_{k}-E_{s}-E_{\mathrm{em}}-E_{g}
$$

Then the Lagrange equation formally is

$$
\frac{\mathrm{d}}{\mathrm{dt}}\left(\frac{\partial L}{\partial \dot{h}}\right)-\frac{\partial L}{\partial h}=0 .
$$


Replacing the expressions of $E_{k}, E_{s}, E_{\text {em }}$ and $E_{g}$ obtained respectively in (4), (5), (8) and (9) in (10) and (11) yields the final equation governing the free surface displacement $h(t)$

$$
\ddot{h}\left(\frac{2}{3}+\frac{V}{4 h^{3}}\right)-\frac{3}{8} \dot{h}^{2} \frac{V}{h^{4}}+g-\frac{2 \gamma}{\rho} \frac{1}{h^{2}}-\frac{1}{8} \sigma \omega B_{0}^{2} \sin (2 \omega t) \frac{V}{h^{2}}=0 .
$$

Equation (12) may be rewritten by using a non-dimensional time $t_{+}=\omega t$. We then obtain another expression of equation (12) which put forth the electromagnetic interaction parameter $N$, namely

$$
\ddot{h}\left(\frac{2}{3}+\frac{V}{4 h^{3}}\right)-\frac{3}{8} \dot{h}^{2} \frac{V}{h^{4}}+\frac{g}{\omega^{2}}-\frac{2 \gamma}{\omega^{2} \rho} \frac{1}{h^{2}}-\frac{1}{8} N \sin \left(2 t_{+}\right) \frac{V}{h^{2}}=0,
$$

with $N=\frac{\sigma B_{0}^{2}}{\rho \omega}$ being the non-dimensional interaction parameter.

Equation (13) contains an electromagnetic forcing term which oscillates at twice the magnetic field frequency.

2. Static equilibrium. Without any magnetic field, the liquid drop is at rest. The static equilibrium, which is readily obtained from equation (13) with $N=0$, yields the height $h_{0}$ of the pool

$$
h_{0}=\left(\frac{2 \gamma}{\rho g}\right)^{1 / 2} \text {. }
$$

The initial height $h_{0}$ is simply related to the gravito-capillary length. That value is in accordance with the exact two-dimensional solution and may also be obtained by means of a simple balance between the hydrostatic pressure and the surface tension at the edge of the pool. Note that for a mercury pool, the numerical value of $h_{0}$ is $2.74 \mathrm{~mm}$ with $\rho=13590 \mathrm{~kg} / \mathrm{m}^{3}$ and $\gamma=0.5 \mathrm{~N} / \mathrm{m}$. That value is quite close to the experimental observation [1].

3. Case of small deformations. For a physical interpretation, it is of interest to consider first small-amplitude oscillations. This case corresponds to small values of the interaction parameter $N$. Thus, we consider a small departure $\eta$ of the pool height from rest:

$$
h(t)=h_{0}+\eta(t)
$$

where $\eta / h_{0}=O(N), N$ being a small parameter.

We shall also use the hypothesis $h_{0}<<a_{0}, a_{0}=30 \mathrm{~mm}$ being the pool radius at rest, and neglect the corresponding terms.

Introducing (15) in equation (13), linearizing and retaining only the term in $O(N)$ yields the equation governing the drop motion

$$
\ddot{\eta}+\frac{\Omega^{2}}{\omega^{2}} \eta=\frac{1}{2} N \sin \left(2 t_{+}\right) h_{0}
$$

where

$$
\Omega^{2}=8 \sqrt{2}\left(\frac{\gamma g}{\rho a_{0}^{4}}\right)
$$

The amplitude $\eta$ is governed by an electromagnetically-forced oscillator equation. The dimensional eigenfrequency of the free oscillations is $F=\Omega /(2 \pi)$. The solution of equation (16) is readily obtained

$$
\eta=N \frac{\omega^{2} \sin \left(2 t_{+}\right)}{2\left(\Omega^{2}-4 \omega^{2}\right)} h_{0} .
$$




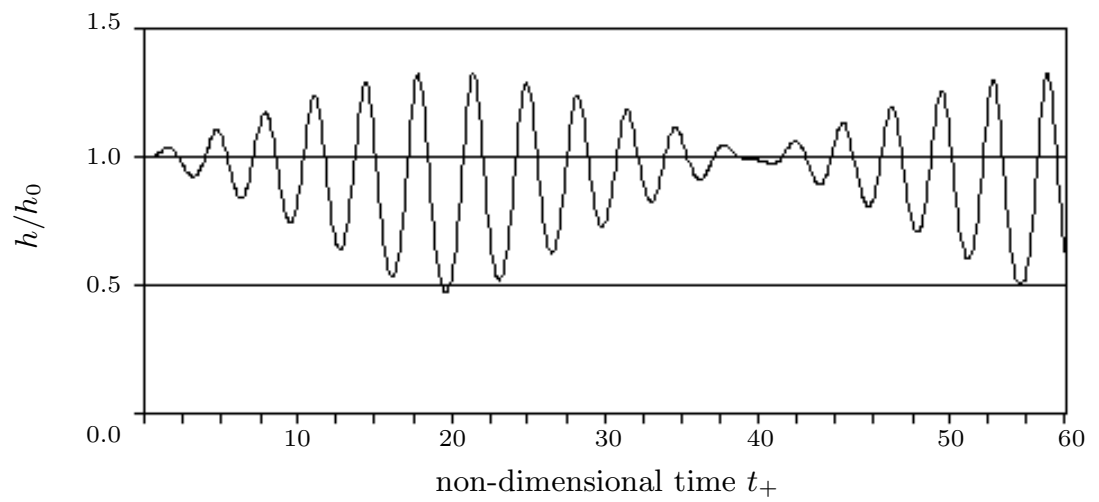

Fig. 3. Time evolution of the free surface height $h(t)$ for $N=0.1$ and $f=1.2 \mathrm{~Hz} ; h(t)$ is normalised by the surface height at rest $h_{0} ; t_{+}=\omega t$.

Solution (18) exhibits a resonance point when the magnetic field frequency $f$ is equal to $F / 2$. The amplitude oscillates at the same frequency as that of the electromagnetic forces, i.e., $2 f$. The existence of a singularity is nothing but a consequence of the absence of any damping or non-linear interactions. In the case of a mercury pool already considered in the previous Section, the numerical value of $F$ is $2.46 \mathrm{~Hz}$ with $a_{0}=30 \mathrm{~mm}$.

4. Finite amplitude oscillations. Let us consider the general case, where the interaction $N$ has a finite value. The free surface height $h(t)$ is governed by a non-linear ordinary differential equation. That equation may be solved numerically by considering the initial value problem with the following initial conditions:

$$
h(0)=h_{0} \quad \text { and } \quad \dot{h}(0)=0 .
$$

A fourth-order Runge-Kutta method has been presently used, and the results are shown in Figs. 3 and 4. The time evolution of $h(t)$ in Fig. 3 exhibits oscillations at

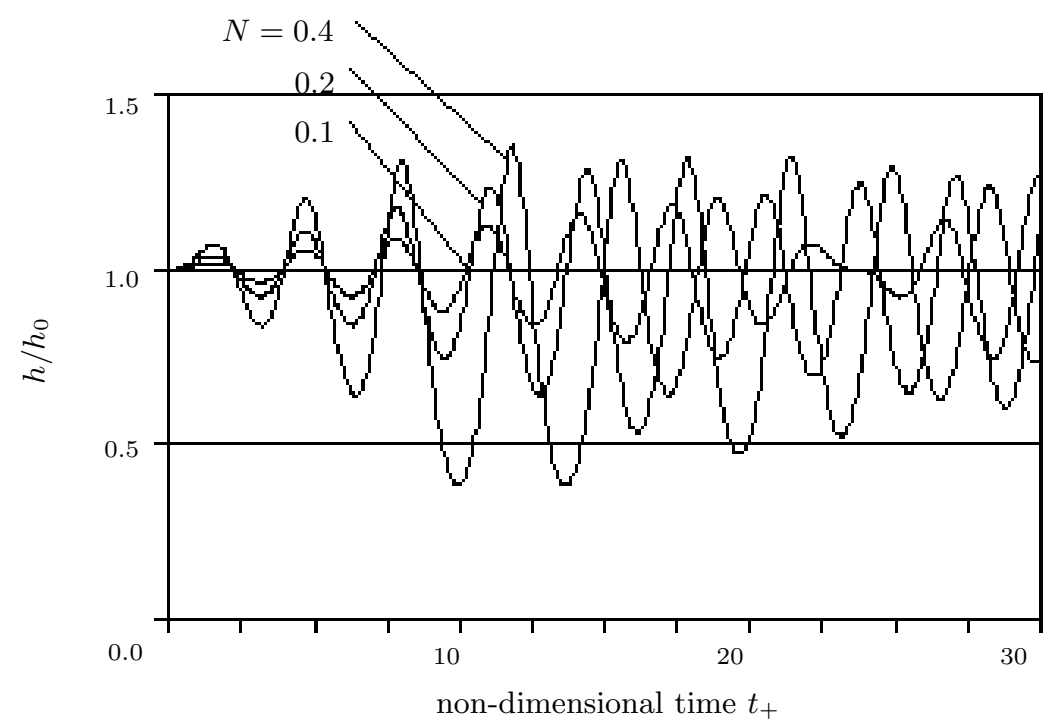

Fig. 4. Time evolution of the free surface height $h(t)$ for various values of the interaction parameter $N$ and $f=1.2 \mathrm{~Hz} ; h(t)$ is normalised by the surface height at rest $h_{0} ; t_{+}=\omega t$. 
twice the magnetic field frequency as in the linear case (cf. equation (18)). However, non-linear interactions are responsible for a slow modulation of the amplitude (Fig. 3). The maximum value of $h(t)$ increases with the interaction parameter $N$, as shown in Fig. 4. However, the computations show that when the value of $N$ is greater than 0.5 approximately, the minimum value of $h(t)$ becomes negative. That means that beyond a magnetic field strength the layer thickness tends to zero and the drop breaks out. Regarding the experiments [1], the axisymmetric regime exists only for weak values of the interaction parameter, typically $N \leq 0.1$. Beyond that value there appear non-symmetric modes, which are triggered by a parametric-type instability [1].

5. Conclusions. The oscillatory motion of a liquid metal drop submitted to a low-frequency alternating magnetic field has been analysed. The analysis has been restricted to the first axisymmetric deformation, where the top free surface remains horizontal. An overall approach using the Lagrange equations has been used. We have put forth that the Lagrange method is quite easy to apply in the present problem. The free surface amplitude equation has been easily obtained once the Lagrangian was determined. Note that the existence of a Lagrangian function in the present low-frequency case is related to the fact that the electromagnetic forced are of gradient type [4]. It is then possible to define an electromagnetic force potential. The drawback of the method lies in the fact that only a simple pool geometry may be considered in order to limit the calculation difficulties. The future prospect is to extend the present analysis to non-symmetric free surface deformations which appears when the magnetic field is sufficiently large.

\section{REFERENCES}

1. S. Daugan, Y. Fautrelle, J. Etay. Free surface horizontal waves generated by low frequency alternating magnetic fields. In: Proc. of the TMS Symposium on Fluid-Flow Phenomena in Metals Processing (San Diego, USA, 28 Feb.-4 March, 1999), Ed. by TMS Pub. (Warrendale PA), pp. 41-48.

2. F. Ingwiller, F. Bonnel, Y. Fautrelle, S. Daugan, J. Etay. Dynamique d'une goutte de métal liquide soumise á un champ magnétique alternatif. In: Proc. of the 9th Colloque Francophone FLUVISU 2001 (Rouen, France, June 2001), CORIA Ed., p. $129-134$

3. J.M. Galpin, Y. Fautrelle, A. Sneyd. Parametric instability in low frequency magnetic stirring. J. Fluid Mech., vol. 239 (1992), pp. 409-427.

4. Y. Fautrelle, A. Sneyd. Magnetic stirring at low frequency magnetic field. J. Fluid Mech., (2003) (in press.) 